\title{
Monte Carlo Simulation of the Director Field of a Nematic Liquid Crystal with Three Elastic Coefficients
}

\author{
T. Gruhn and S. Hess \\ Institut für Theoretische Physik, Technische Universität Berlin, D-10623 Berlin, Germany
}

Z. Naturforsch. 51 a, 1-9 (1996); received December 15, 1995

\begin{abstract}
In this article, a Monte Carlo simulation is presented, which generates the equilibrium director field of a nematic liquid crystal under the influence of an external field and fixed boundary conditions. The liquid crystal is characterized by a set of directors on a spatially fixed lattice. The simulation is based on an expression for the Frank free energy with three elastic coefficients. The chosen discretisation conserves the nematic symmetry, which means $\boldsymbol{n}$ and $\boldsymbol{- n}$ are equivalent. The results for several Frederiks geometries with homogeneous and spatially modulated external fields are shown, as well as an investigation of a capillary with homeotropic boundary conditions. Further we compare our method with the Lebwohl-Lasher model and introduce an extension of the latter which distinguishes between splay-, twist- and bend-configurations.
\end{abstract}

\section{Introduction}

The orientation of a nematic liquid crystal can be described by a director field $\boldsymbol{n}(\boldsymbol{x})[1,2]$. The equilibrium state of the orientation field is characterized by a minimum of the Frank free energy [3]. The knowledge of the director field is of great interest for theoretical problems as well as for technical applications [1, 2].

Though the Monte Carlo simulation is a well established method to determine equilibrium properties in high dimensional state spaces, to our knowledge no one has ever applied the Monte Carlo technique to the Frank free energy of a director field: On one hand simulations were performed which use relaxation equations, inlcuding derivatives of the Frank free energy, on the other hand there are Monte Carlo investigations based on the Lebwohl-Lasher model, which is derived from a microscopic approach. In our Monte Carlo method the acceptance probability for a variation of the director lattice, which describes the orientation field, depends on the Frank free energy difference. After a number of variation steps the equilibrium director field occurs. This also works well near second order phase transition points.

Our approach allows the free choice of the elastic coefficients $K_{1}, K_{2}, K_{3}$. To simplify the calculations, they are often assumed to be equal (one-coefficient approximation) [1, 2], but for real materials one finds various elastic coefficient ratios: MBBA at $23^{\circ} \mathrm{C}$ reveals $K_{3} / K_{1}=1.4$ and $K_{3} / K_{2}=2.2$ [4] and PAA at

Reprint requests to Prof. S. Hess, e-mail:

S.Hess@ physik.tu-berlin.de, T.Gruhn@physik.tu-berlin.de. $120^{\circ} \mathrm{C}$ has $K_{3} / K_{1}=1.9$ and $K_{3} / K_{2}=3.0$ [5], further there are alkenyl liquid crystals with $K_{3} / K_{1}=0.5$ [6] and for discotic nematics - for which our simulation is valid as well - ratios of $K_{3} / K_{2}<1$ have been estimated [7].

In nematic liquid crystals the director $\boldsymbol{n}$ is physically equivalent to $-\boldsymbol{n}$, which is a necessary requirement for the description of $s=1 / 2$ disclinations [1,2]. Our simulation starts from a tensorial expression of the Frank free energy, and therefore the nematic symmetry is conserved (compare [8]).

In Sect. 2 we derive the expression for the discretized Frank free energy in the presence of an external field and describe the Monte Carlo algorithm to find equilibrium configurations. Section 3 describes the application of the algorithm to several Frederiks cells with homogeneous and inhomogeneous external fields and the results for the simulation of a capillary with homeotropic boundary conditions. In Sect. 4 we compare our algorithm with the Lebwohl-Lasher model. We obtain an analogy between the LebwohlLasher model and our method in the one-coefficient approximation and introduce an extended pseudo-microscopic model, including energy expressions that correspond to the discretized splay-, twist- and benddeformation energies.

\section{Derivation of the Algorithm}

\subsection{Theoretical Background}

In the following sections, we describe a Monte Carlo technique that produces equilibrium configura- 
tions of the orientation field of a nematic liquid crystal which is deep within the nematic regime, such that the degree of order is practically constant.

In general, the orientation distribution of uniaxial molecules can be described by the second rank alignment tensor [9]

$$
a_{\mu v}(x, t)=\sqrt{\frac{15}{2}}\left\langle\overleftrightarrow{\mathrm{u}}_{\mu} \overrightarrow{\mathrm{u}}_{\mathrm{v}}\right\rangle,
$$

where $\boldsymbol{u}$ is a unit vector parallel to the molecules' figure axes and the brackets \langle\rangle indicate an average. The Greek subscripts refer to Cartesian components, for which the summation convention is used, and $\overleftrightarrow{\mathbf{b}}$ denotes the symmetric-traceless part of a tensor $\mathbf{b}$ :

$$
\overleftrightarrow{\mathrm{b}_{\mu \nu}}=\frac{1}{2}\left(b_{\mu v}+b_{v \mu}\right)-\frac{1}{3} b_{\lambda \lambda} \delta_{\mu v} .
$$

We assume a uniaxial orientation of the molecules, which is distributed symmetrically around a director $\boldsymbol{n}(\boldsymbol{x})$; then, the alignment tensor becomes

$$
a_{\mu v}=\sqrt{\frac{3}{2}} a \overleftrightarrow{\mathrm{n}_{\mu} \mathrm{n}_{v}}
$$

Here we have $a=\sqrt{5} S$ with $S=\left\langle P_{2}\left(\mathbf{u}_{\mu} \mathbf{n}_{\mu}\right)\right\rangle$ being the Maier-Saupe order parameter [10]. If we assume $S$ to be constant for the whole system, all we need to describe the orientation of the liquid crystal is $\overleftrightarrow{\mathbf{n}}(\boldsymbol{x})$.

For equilibrium configurations of the director field the Frank free energy $F_{a}=\int_{V} f_{a} \mathrm{~d}^{3} r$ is in its absolute minimum (compare [11]). The energy density $f_{a}$ can be divided into $f_{a}=f_{\mathrm{LdG}}+f_{\mathrm{or}}, f_{\mathrm{or}}=f_{\text {field }}+f_{\mathrm{el}}$. The Landau-de Gennes part [12] $f_{\text {LdG }}$ is irrelevant here, because it only depends on $S$, which was assumed to be constant. The influence of an orienting external field is described by $f_{\text {field }}$. For a magnetic field $\boldsymbol{B}$ it becomes [2]:

$$
f_{\text {field }}=-\frac{1}{2} \mu_{0}^{-1} \chi_{a}\left(n_{v} B_{v}\right)^{2},
$$

where $\mu_{0}$ is the vacuum magnetic susceptibility, and $\chi_{a}$ is given by $\chi_{a}=S\left(\chi_{\|}-\chi_{\perp}\right)$, where $\chi_{\|}$and $\chi_{\perp}$ are the relative magnetic susceptibilities, parallel and perpendicular to the molecule axis of a perfectly ordered liquid crystal. The elastic energy density $f_{\mathrm{el}}$ arises from deformations of the director field. Generally, the bulk contribution to the elastic energy consists of three terms, associated with splay, twist and bend deformations:

$$
\begin{aligned}
f_{\mathrm{el}}= & \frac{1}{2}\left[K_{1}(\nabla \cdot \boldsymbol{n})^{2}+K_{2}(\boldsymbol{n} \cdot(\nabla \times \boldsymbol{n}))^{2}\right. \\
& \left.+K_{3}(\boldsymbol{n} \times(\nabla \times \boldsymbol{n}))^{2}\right] .
\end{aligned}
$$

Assuming that the director field is homogeneous in one direction, we describe the director field by a finite set of directors $\boldsymbol{n}(i, j)$ sitting on a twodimensional rectangular lattice.

It turns out that a straight forward discretisation of the spatial derivatives in (3) leads to a loss of the head-tail-symmetry, which means that $\boldsymbol{n}$ is no longer equivalent to $-\boldsymbol{n}$. This already occurs in the one coefficient approximation $\left(K_{1}=K_{2}=K_{3}\right)$, which is discussed in [13]. Therefore, we have used another expression for the elastic energy density, which is - except for surface terms, disregarded here - algebraically equivalent to (3):

$$
\begin{aligned}
f_{\mathrm{el}}=\frac{1}{2}[ & \frac{1}{2} \\
& K_{2}\left(\nabla_{\lambda} n_{\mu} n_{\mathrm{v}}\right)\left(\nabla_{\lambda} n_{\mu} n_{\mathrm{v}}\right) \\
& +\left(K_{1}-K_{2}\right)\left(\nabla_{\lambda} n_{\lambda} n_{\mu}\right)\left(\nabla_{\mathrm{v}} n_{\mathrm{v}} n_{\mu}\right) \\
& \left.+\frac{1}{2}\left(K_{3}-K_{1}\right)\left(n_{\mu} n_{\mathrm{v}}\right)\left(\nabla_{\mu} n_{\lambda} n_{\mathrm{\kappa}}\right)\left(\nabla_{\mathrm{v}} n_{\lambda} n_{\mathrm{k}}\right)\right] .
\end{aligned}
$$

In this form, the director always appears as a dyad $\boldsymbol{n} \boldsymbol{n}$; thus, the nematic symmetry still exists after the discretisation.

The last term in (4) demands that the discretisation of the derivative expressions are defined on the director lattice points. We analyzed all sensible symmetrical discretisation schemes for first order derivatives and found that none of them is suitable for the Monte Carlo technique [14]. The reason is that they do not involve differences of nearest neighbor values and thus lead to uncoupled sublattices. Therefore, we used the following discretized energy density for our simulation:

$$
\begin{aligned}
f_{\text {or }}^{*}(i, j): & =\frac{1}{4} \sum_{r= \pm 1} \sum_{s= \pm 1}\left[\frac{1}{2} K_{2}^{*} \sum_{\mu, v=1}^{3} \sum_{\lambda=1}^{2}\left(\mathscr{D}_{\lambda \mu \nu}^{(r, s)}(i, j)\right)^{2}\right. \\
& +\left(K_{1}^{*}-K_{2}^{*}\right) \sum_{v=1}^{3}\left(\sum_{\lambda=1}^{2} \mathscr{D}_{\lambda \lambda v}^{(r, s)}(i, j)\right)^{2} \\
& \left.+\frac{1}{2}\left(K_{3}^{*}-K_{1}^{*}\right) \sum_{\mu, v=1}^{3}\left(\sum_{\lambda=1}^{2} n_{\lambda}(i, j) \mathscr{D}_{\lambda \mu \nu}^{(r, s)}(i, j)\right)^{2}\right] \\
& +\left(\frac{\pi}{N+1} B^{*}(i, j)\right)^{2}\left(\sum_{v=1}^{3} n_{v}(i, j) \hat{B}_{v}(i, j)\right)^{2} \cdot(5)
\end{aligned}
$$

Here the elastic part of $f_{\text {or }}^{*}$ is defined as the arithmetic average of four expressions for $f_{\mathrm{el}}^{*}$, built with the four asymmetrical discretisations of the derivatives (see Figure 1):

$$
\begin{array}{r}
\mathscr{D}_{1 \mu \mathrm{v}}^{(r, s)}(0,0):=n_{\mu} n_{\mathrm{v}}(r, 0)-n_{\mu} n_{\mathrm{v}}(0,0), \\
(r, s \in\{1,-1\}), \\
\mathscr{D}_{2 \mu \mathrm{v}}^{(r, s)}(0,0):=n_{\mu} n_{\mathrm{v}}(0, s)-n_{\mu} n_{\mathrm{v}}(0,0), \\
(r, s \in\{1,-1\}) .
\end{array}
$$

The dimensionless quantities in (5), which are denoted with an asterisk, will be defined in Section 3.2. In the one coefficient approximation $K:=K_{1}$ 


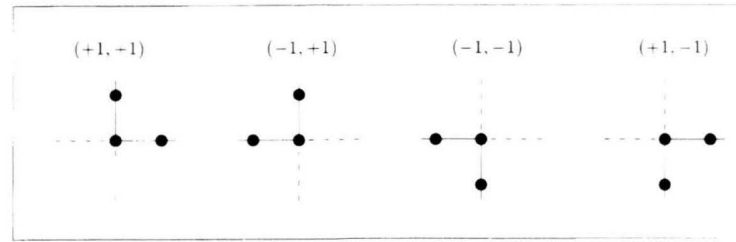

Fig. 1. The discretized elastic energy density is the arithmetic average of four $f_{\mathrm{el}}$ terms, built with the four asymmetrical discretisations $\mathscr{D}_{\lambda \mu \mathrm{v}}^{(r, s)}$ with $(r, s) \in\{(+1,+1)$, $(-1,+1),(-1,-1),(+1,-1)\}$.

$=K_{2}=K_{3}$ the elastic part of (5) becomes the much simpler expression

$f_{\mathrm{el}}^{*}(i, j)=\frac{1}{2} K^{*} \sum_{\|(i, j)-(k, l)\|=1}\left[1-\left[\sum_{\mu=1}^{3} n_{\mu}(i, j) n_{\mu}(k, l)\right]^{2}\right]$.

For the extension to threedimensional simulations one has to calculate the average of eight Frank free energy expressions, built with the different asymmetrical discretisations of $\nabla_{\mu} n_{\lambda} n_{\kappa}$.

\subsection{Monte Carlo Method}

The Monte Carlo simulation is a well established method, which can be used to calculate the ensemble average of an observable $a$ in a state space $G$ : $\langle a\rangle=\int_{G} a(\Gamma) f(\Gamma) \mathrm{d} \Gamma$ with a probability density

$$
f(\Gamma)=\frac{e^{-\frac{F(\Gamma)}{k T}}}{\int_{G} e^{-\frac{F(\Gamma)}{k T}} \mathrm{~d} \Gamma}
$$

for a state $\Gamma$ which has the energy $F(\Gamma)$ [15]. The basic idea of the Monte Carlo method is to generate a chain of configuration states $\Gamma_{k}$ with a probability given by $f\left(\Gamma_{k}\right)$. Then one finds

$$
\langle a\rangle=\lim _{m \rightarrow \infty} \frac{1}{m} \sum_{k=1}^{m} a\left(\Gamma_{k}\right),
$$

where the prime on the summation sign denotes that the $\Gamma_{k}$ are distributed over $G$ with $f\left(\Gamma_{k}\right)$.

Now we look at our director lattice system and apply the Metropolis algorithm [16] to produce a chain of configuration states with the mentioned distribution:

- Create a random order in which the directors shall be treated.

- Choose randomly whether the actual director shall be rotated around the $x$-, the $y$ - or the $z$-axis.
- Rotate the director by a random angle $\psi$, uniformly distributed over the interval $\left[-\psi_{\mathrm{m}}, \psi_{\mathrm{m}}\right]$, where $\psi_{\mathrm{m}}$ is a fixed, suitably chosen maximum rotation angle.

- Calculate the energy difference $\Delta F(\boldsymbol{n} \rightarrow \tilde{\boldsymbol{n}})$ between the configuration with the old director $\boldsymbol{n}$ and the new one $\tilde{\boldsymbol{n}}$.

- Accept the new director with the probability $p(\boldsymbol{n} \rightarrow \tilde{\boldsymbol{n}})=\min \{1, \exp [-\Delta F / k T]\}$. If the new director is rejected, retain the old director $\boldsymbol{n}$.

- Try to change the next director. If all directors have been treated, one Monte Carlo step is made and for the next step another random sequence of lattice points must be chosen.

After a number of equilibration steps the algorithm calculates via (8) the mean equilibrium orientation field $\left\langle n_{\mu} n_{v}\right\rangle\left(\boldsymbol{x}_{i}\right)$.

\section{Applications}

\subsection{Frederiks Cells}

Frederiks cells are thin confined liquid crystal films with given director orientations at the surfaces [2]. Under the influence of an external field $\boldsymbol{B}$, which is perpendicular to the director field, one observes a second order phase transition at a critical field strength $B_{\text {crit }}$ : For an increasing $B$, the director field remains unchanged as long as $B<B_{\text {crit }}$; above $B_{\text {crit }}$, it becomes more and more parallel to the $\boldsymbol{B}$-direction. Figure 2 shows various Frederiks geometries with $B>B_{\text {crit }}$. For the splay-, twist- and bend-geometries (Fig. 2.1-2.3) the respective $B_{\text {crit }}$ depend on one elastic

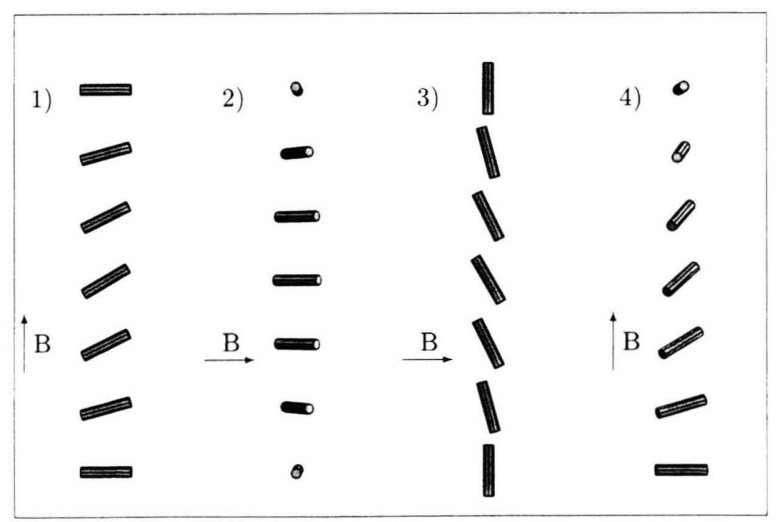

Fig. 2. Director fields for the Frederiks cells above the critical field strength: 1) splay geometry, 2) twist geometry, 3) bend geometry, 4) Schadt-Helfrich cell. 
coefficient each:

$$
B_{\mathrm{crit}}^{(i)}=\frac{\pi}{d} \sqrt{\frac{K_{i} \mu_{0}}{\chi_{a}}} .
$$

The Schadt-Helfrich-cell (Fig. 2.4) includes all three elastic deformations and therefore its critical field strength depends on all three elastic coefficients:

$$
B_{\mathrm{crit}}^{\mathrm{SH}}=\frac{\pi}{d} \sqrt{\frac{\mu_{0}}{\chi_{a}}\left[K_{1}+\left(K_{3}-2 K_{2}\right)\left(\varphi_{\max } / \pi\right)^{2}\right] .}
$$

(More general expressions for the threshold values are listed in [17]). Since there are analytical solutions for the Frederiks cells, they are good objects to test our algorithm.

\subsection{Technical Details}

In this section we present some technical details of our program. To describe the various Frederiks cells, we choose a lattice of $N_{x} \times N_{y}$ directors having a distance $l$ to their neighbors. The system has periodic boundary conditions in the $x$-direction and fixed boundary directors $\boldsymbol{n}(x, 0), \boldsymbol{n}\left(x, N_{y}+1\right)$ in the $y$-direction. This represents a liquid crystal layer of the height $d=\left(N_{y}+1\right) l$. We assume a director field, which is homogeneous in the $z$-direction: So every director in our lattice represents the orientation of an $l \times l \times b$ box, where $b$ is the $z$-extension of the observed liquid crystal volume. While the order of magnitude for $l$ is about $1 \mu \mathrm{m}, b$ is circa eighty times larger (see below). The dimensionless quantities of (5) are defined by the following equations:

$$
\begin{array}{ll}
K_{i}=K_{i}^{*} K, \quad(i=1,2,3), & F_{\text {or }}=\frac{b}{2} K F_{\text {or }}^{*}, \\
B_{\mu}=B \hat{B}_{\mu}, & f_{\text {or }}=\frac{K}{2 l^{2}} f_{\text {or }}^{*}, \\
B=\left(\frac{\pi}{\left(N_{y}+1\right) l} \sqrt{\frac{K \mu_{0}}{\chi_{a}}}\right) B^{*}, & T=\left(k_{B}^{-1} \frac{b}{2} K\right) T^{*} .
\end{array}
$$

It was our purpose to choose the run parameters of the simulation such that it works well for field strengths $B$ close to $B_{\text {crit }}$.

The simulations were carried out at the dimensionless temperature $T^{*}=2 \cdot 10^{-5}$ with a maximum rotation angle of $\psi_{\mathrm{m}}=0.5^{\circ}$. With a mean elastic coefficient $K=5 \cdot 10^{-12} N$ and a width $b=85 \mu \mathrm{m}$, our $T^{*}$ corresponds to a temperature $T \approx 300 \mathrm{~K}$.
Our system consisted of $15 \times 15$ directors not including the directors needed for the fixed boundary conditions. We took $10^{5}$ equilibration steps and the same number of steps for averaging. To perform $10^{5}$ Monte Carlo steps, the Hewlett/Packard 9000/755 needed about 20 minutes for the one coefficient approximation and 120 minutes with three elastic coefficients, where one Monte Carlo step means that every lattice point is treated once. To save computation time most of the results for the homogeneous Frederiks cells were carried out on a one dimensional lattice, after we had checked in several cases that the two dimensional simulation leads to the same director field, being homogeneous along the added dimension.

\subsection{Frederiks Cells with a Homogeneous External Field}

In this section we discuss the simulation results for several Frederiks cells with fixed boundary conditions in the $y$-direction.

Though the Frederiks threshold for the splay geometry only depends on $K_{1}$ the director field for $B>B_{\text {crit }}$ also varies with $K_{3} / K_{1}$ (see [2]). Figure 3 shows the angle $\theta(y)$ between the director $\boldsymbol{n}(y)$ and the easy axis for $B=1.4 B_{\text {crit }}$ and ratios of $K_{3} / K_{1}$ between 0.5 and 2.0. The simulated director fields are symmetrical and agree well with the theoretical curves. One finds that the simulation values for $\theta(y)$ lie about 2 percent above the correct distributions. This is due to a discretisation error, which stems from the low number of director points. For simulations with 30 directors in the $y$ direction the deviation went down to less than 1 percent. All the simulation results for the splay geometry were independent of a variation of $K_{2}$. The analytic results for the splay geometry can be transferred to the bend geometry by exchanging $\theta$ with $90^{\circ}-\theta$ and $K_{1}$ with $K_{3}$. All our simulation tests with the bend geometry were in agreement with this fact. In Fig. 4 the maximum angle $\theta_{\max }$ between the director field and the easy axis is shown for field strength between $B / B_{\text {crit }}=0.5$ and $B / B_{\text {crit }}=2.0$ and for several ratios of $K_{3} / K_{1}$.

Here, again the systematic deviation of about 2 percent from the theoretical $\theta_{\max }$ curves occurs, but nevertheless a value for $B_{\text {crit }}$ can be extracted, which is only about 0.2 percent lower than the correct critical field strength. To illustrate this fact, Fig. 5 shows the simulation results of the maximum angle $\theta_{\max }$ between the director field and the easy axis for twist geometry. Here we focus on the immediate neighborhood of 


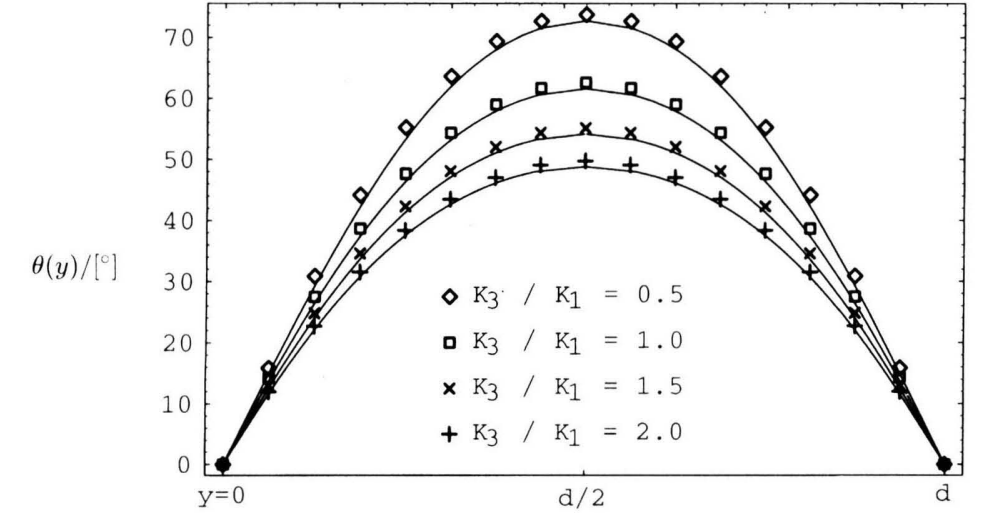

Fig. 3. Tilt angle $\theta(y)$ between the director and the easy axis for the splay geometry with several $K_{3} K_{1}$ ratios at $B^{*}=1.4$. The curves show the analytic solutions. Exchanging $K_{1}$ and $K_{3}$ leads to the solutions for the bend geometry.

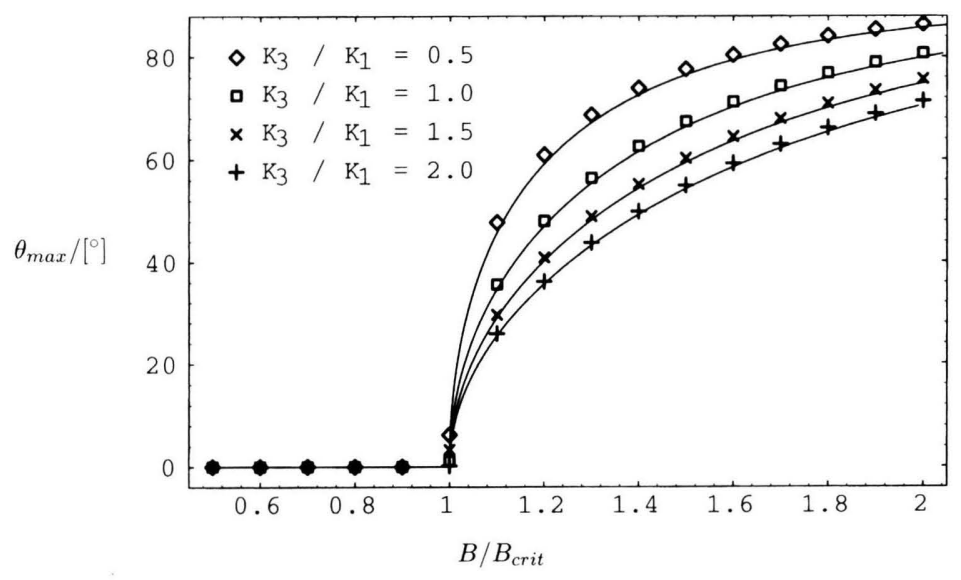

Fig. 4. Maximum tilt angle $\theta_{\text {max }}$ for the splay geometry. The curves show the analytic solutions. Exchanging $K_{1}$ and $K_{3}$ leads to the solutions for the bend geometry.

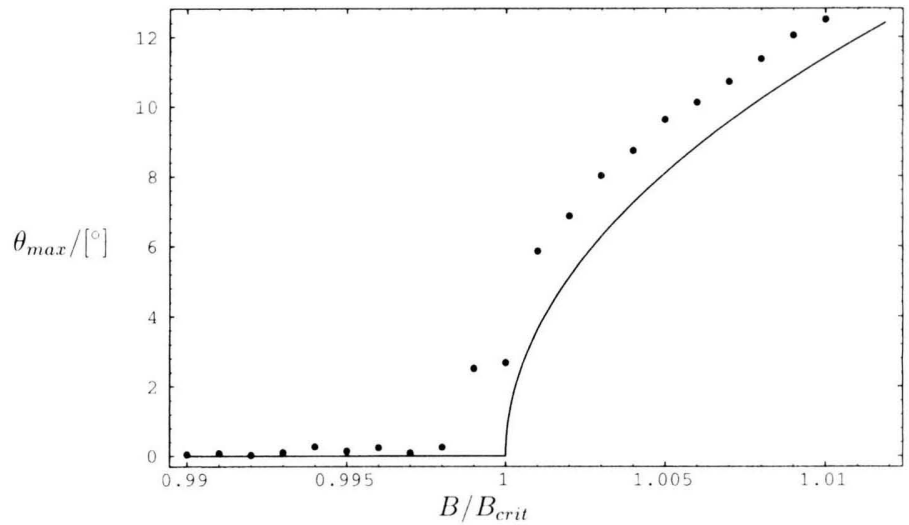

Fig. 5. Maximum tilt angle $\theta_{\max }$ for the twist geometry, focussed on the immediate neighborhood of the Frederiks threshold. The curve shows the analytic solution.

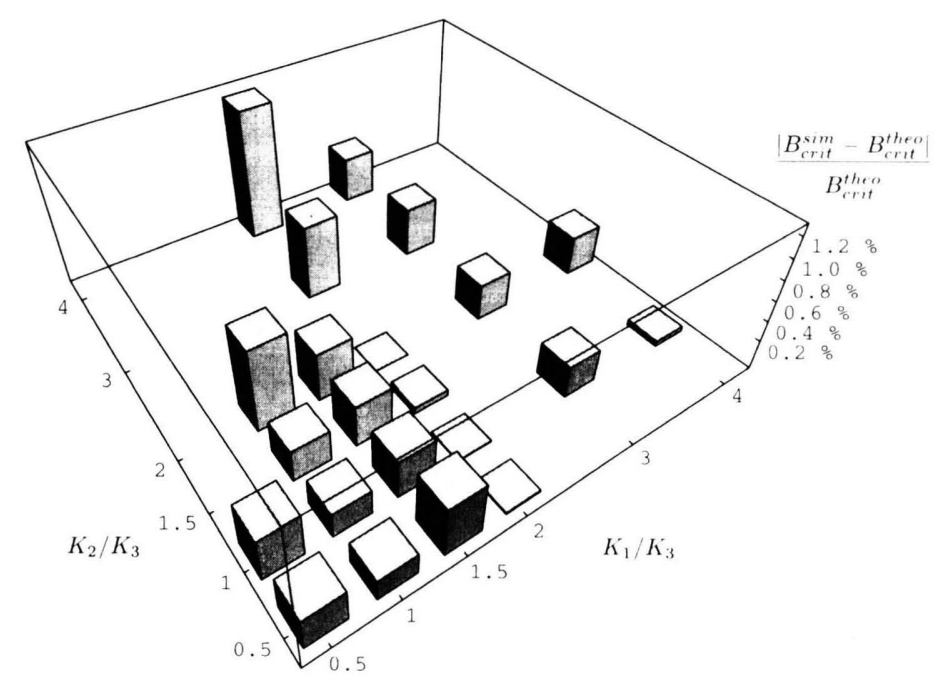

Fig. 6. The simulation was used to estimate the Frederiks thresholds for several $K_{1} / K_{3}$ and $K_{2} / K_{3}$ ratios. The diagram shows the percentual deviations from the analytic solutions. 
$B_{\text {crit }}$. Neglecting the value for $B / B_{\text {crit }}=1.0$ one finds all the evaluated angles on a smooth curve, which can be found by shifting the theoretical curve about $0.002 B_{\text {crit }}$ to the left. The final test was the calculation of the critical field strength $B_{\text {crit }}^{\mathrm{SH}}$ for the SchadtHelfrich cell with perpendicular boundary conditions. This was done, by analysing the maximum tilt angle of the director field. The algorithm test was performed for several values of $K_{1} / K_{3}$ and $K_{2} / K_{3}$ between 0.5 and the rather extreme value of 4.0. Figure 6 shows the relative errors of the simulation results with respect to the theoretical values. For almost every investigated ratio of $K_{1} / K_{3}$ and $K_{2} / K_{3}$ this discretisation error amounts to a few tenths of a percent.

\subsection{Frederiks Cells with an \\ Inhomogeneous External Field}

After the accuracy test with the homogeneous Frederiks cells we simulated a Frederiks cell whose external field $B$ was defined as a mean field strength $B_{\mathrm{m}}$ modulated by a sine function:

$$
B^{*}(x):=B_{\mathrm{m}}^{*}+0.5 \cdot \sin \left(x \cdot \frac{2 \pi}{N_{x}}\right) .
$$

First we describe the simulation results for the one coefficient approximation. The tilt angle field $\theta(x, y)$ for $B_{\mathrm{m}}^{*}=1.1$ is presented in Figure 7. Figure 8 shows the tilt angle in the middle of the cell $\theta(x, d / 2)$ for various mean field strengths between $B_{\mathrm{m}}^{*}=0.8$ and $B_{\mathrm{m}}^{*}=1.2$. The progress of $\theta(x, d / 2)$ for the several $x$-values agrees qualitatively with the curve shown in Figure 5. Because of the coupling in $x$-direction, there is a common critical field strength $\left(B_{\mathrm{m}}\right)_{\text {crit }}^{*}$ for all $x$-values. We found this critical field strength to be $\left(B_{\mathrm{m}}\right)_{\text {crit }}^{*}$ $=0.88 \pm 0.02$, which is lower than the Frederiks threshold $\left(B_{\text {hom }}\right)_{\text {crit }}^{*}=1.0$ for homogeneous $B$-field.

Finally we analyzed how the splay geometry with the sine-modulated external field (11) depends on the $K_{3} / K_{1}$-ratio. Since there are no twist deformations in this cell, the director field is independent of $K_{2}$. Figure 9 shows the difference between the tilt angles

$$
\Delta \theta:=\theta\left[K_{3} / K_{1}=0.5\right]-\theta\left[K_{3} / K_{1}=1.0\right]
$$

of the equilibrium configurations for $K_{3} / K_{1}=0.5$ and $K_{3} / K_{1}=1.0$. At $B_{\mathrm{m}}=1.5 B_{\text {crit }}$ the directors in the middle of the cell point rather in the $y$-direction $\left(\theta(x, d / 2)>45^{\circ}\right)$; thus, in $x$-direction the splay interaction dominates, which is proportional to $K_{1}$. As a

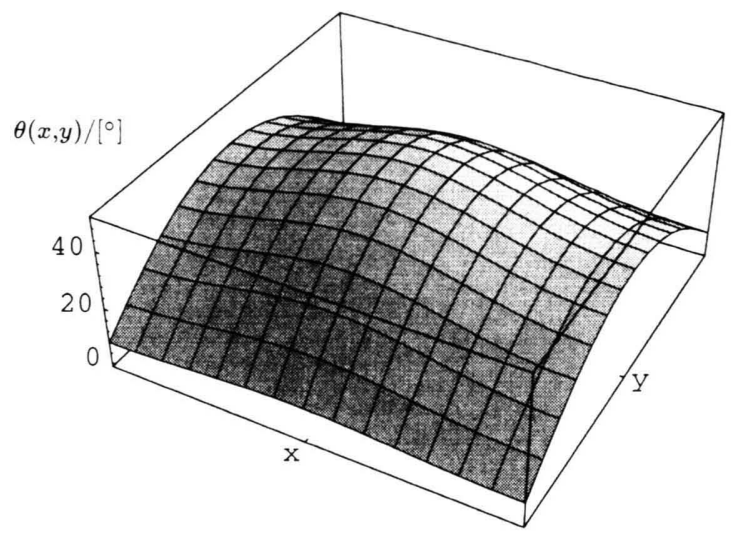

Fig. 7. The equilibrium configuration of the splay geometry with an inhomogeneous external field $B^{*}(x)=1.1+$ $\frac{1}{2} \cdot \sin \left(x \cdot \frac{2 \pi}{N_{x}}\right)$.

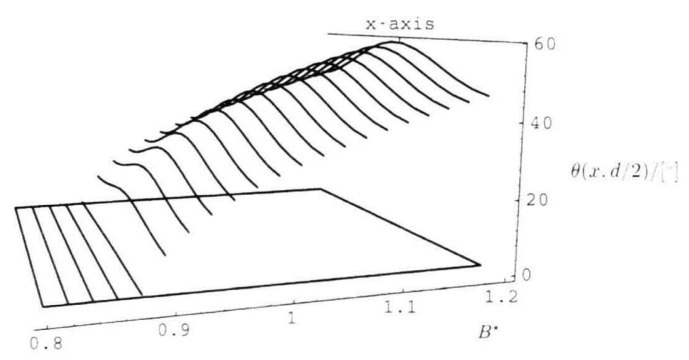

Fig. 8. Tilt angle $\theta(x, d / 2)$ in the middle of a splay geometry with a sine modulated external field $B$.

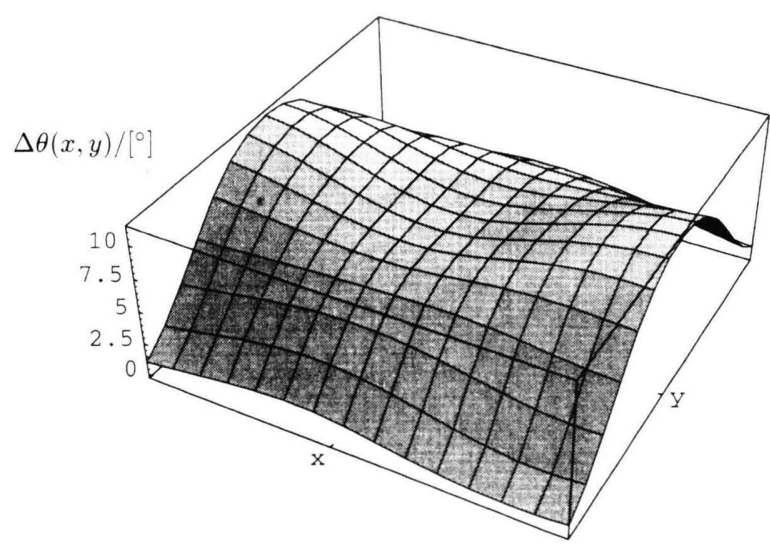

Fig. 9. The figure demonstrates the influence of the $K_{3} / K_{1}$ ratio on the splay geometry with a sine modulated external field. It shows the difference between the tilt angles $\Delta \theta:=\theta\left[K_{3} / K_{1}=0.5\right]-\theta\left[K_{3} / K_{1}=1.0\right]$ for $B_{\mathrm{m}}^{*}=1.5$. 
result, for $K_{3} / K_{1}=0.5$ the directors of the low field strength areas are stronger coupled to the other directors and $\Delta \theta$ becomes especially large.

\subsection{Capillary with Homeotropic Boundary Conditions}

The algorithm presented was constructed such that the nematic symmetries are conserved. Therefore the typical $s=1 / 2$ disclinations can be described. We performed a simulation of a director field $\boldsymbol{n}(x, y)$ in a cylindrical capillary with homeotropic boundary conditions [18], whose symmetry axis lies in $z$-direction. We started with random configurations and ran through a number of $10^{6}$ relaxation steps. To save computation time the simulation was carried out in the one coefficient approximation.

About 5 percent of the simulation runs ended in the escaped structure (Fig. 10), which is the stable configuration with the lowest energy. In the remaining 95 percent the program found a planar configuration with two $s=1 / 2$ disclinations, like the one shown in Figure 11. The latter configuration is metastable, but obviously more likely to arise for our simulation. We assume that high elastic energies in the disclination cores are softened by the discretisation of the director field.

Anyway, since near the disclination the order parameter is not spatially constant anymore, the liquid crystal orientation field is only described correctly with the help of the full alignment tensor a, which was done in [19].

\section{Pseudo-Microscopic Lattice Model}

\subsection{Comparison with the Lebwohl-Lasher Energy}

In the one coefficient approximation the discretized elastic energy $F_{\text {el }}$ reduces to

$$
F_{\mathrm{el}}=\frac{K l}{2} \sum_{\langle i, j\rangle}\left[-\left[n_{\mu}\left(\boldsymbol{x}_{i}\right) n_{\mu}\left(\boldsymbol{x}_{j}\right)\right]^{2}+1\right],
$$

where $\langle i, j\rangle$ denotes a summation over all nearest neighbors. This is very similar to the Hamilton function of the Lebwohl-Lasher model [20]:

$$
H=\frac{3}{2} \varepsilon \sum_{\langle i, j\rangle}\left[-\left[u_{\mu}\left(\boldsymbol{x}_{i}\right) u_{\mu}\left(\boldsymbol{x}_{j}\right)\right]^{2}+\frac{1}{3}\right] .
$$

The parts depending on the orientation have the same form, but (12) is built with the macroscopic director $\boldsymbol{n}$, while (13) depends on the microscopic director $\boldsymbol{u}$,

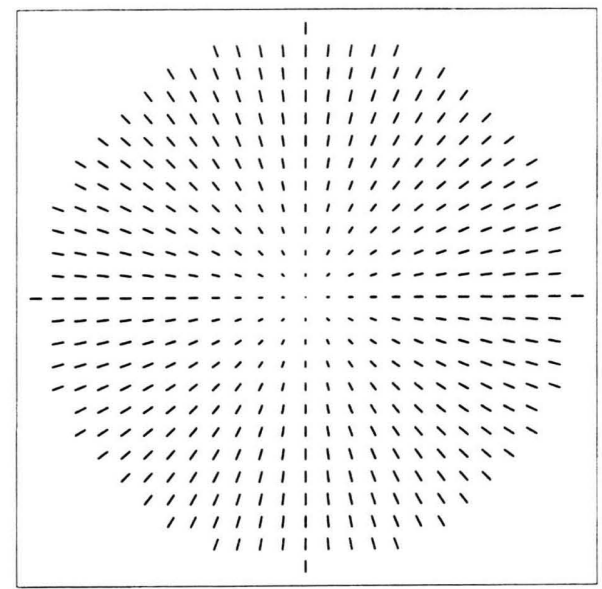

Fig. 10. For a capillary with homeotropic boundary conditions, 5 percent of the simulation runs ended in the escaped solution, which has the lowest elastic energy ...

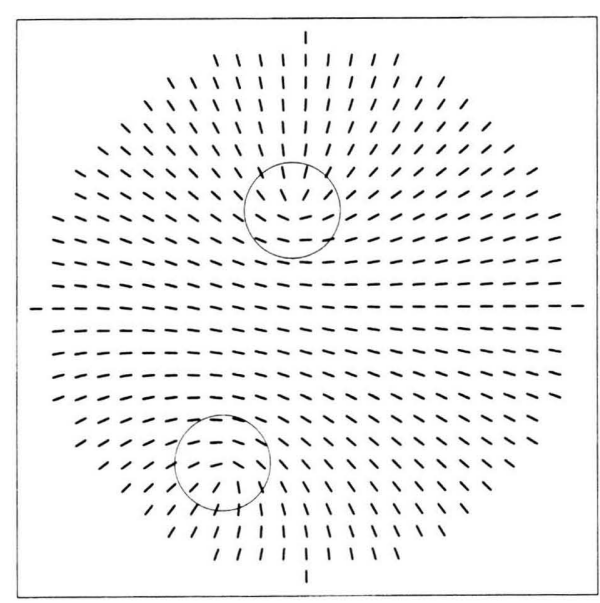

Fig. 11. ... The remaining 95 percent of the simulation runs relaxated to a planar configuration with two $s=1 / 2$ disclinations, similar to the one shown here.

which directly describes the orientation of a single

molecule.

Priest [21] calculated for the Lebwohl-Laser model in molecular-field approximation the relation $\varepsilon=\frac{K l}{3 S^{2}}$, which confirms that $\boldsymbol{n}=\boldsymbol{u}$ is only valid for $S=1$. We obtain that Priest's result is approximately valid for $\left[u_{\mu}\left(\boldsymbol{x}_{i}\right) u_{\mu}\left(\boldsymbol{x}_{j}\right)\right] \approx S\left[n_{\mu}\left(\boldsymbol{x}_{i}\right) n_{\mu}\left(\boldsymbol{x}_{j}\right)\right]$. Cleaver and Allen [22] found for a Lebwohl-Lasher simulation $K / \varepsilon \sim S^{1.76}$. 
Compared with the Lebwohl-Lasher model, our simulation technique has a number of advantages:

- The Lebwohl-Lasher model starts with the assumption that the molecules themselves are located on fixed lattice points, which is hard to motivate. Our simulation is the discretized orientation field, whose discretisation errors are proportional to $l^{2}$.

- The free choice of the lattice point distance $l$ allows our simulation to calculate rather large geometries, while the Lebwohl-Lasher model is restricted to microscopic length scales.

- Our method can treat energy expressions with three (or more) elastic coefficients.

\subsection{An Extended Lebwohl-Lasher Model}

The analogy of the two methods inspired us to define an extended energy expression for the interaction of two molecules, analogously to the discretized three elastic coefficient term. Unfortunately, the expression (5) cannot be divided into pair interactions. Instead of this one can consider a director field which is homogeneous in $y$ - and $z$-direction. Then (5) can be expressed as a sum of pair interaction terms between neighbor directors $\boldsymbol{u}^{a}$ and $\boldsymbol{u}^{b}$ on the $x$-axis, where the interaction terms have the form

$$
\begin{aligned}
E_{a b}= & j_{2}\left[1-\left(u_{\mu}^{a} u_{\mu}^{b}\right)^{2}\right] \\
& +\left(j_{1}-j_{2}\right)\left[u_{1}^{a} u_{\mu}^{a}-u_{1}^{b} u_{\mu}^{b}\right]\left[u_{1}^{a} u_{\mu}^{a}-u_{1}^{b} u_{\mu}^{b}\right] \\
& +\frac{1}{2}\left(j_{3}-j_{1}\right)\left[\left(u_{1}^{a}\right)^{2}+\left(u_{1}^{b}\right)^{2}\right]\left[1-\left(u_{\mu}^{a} u_{\mu}^{b}\right)^{2}\right]
\end{aligned}
$$

Here, $j_{1}, j_{2}$ and $j_{3}$ are arbitrary parameters and $u_{1}^{a}$ (resp. $u_{1}^{b}$ ) denotes the $x$-component of $\boldsymbol{u}^{a}$ (resp. $\boldsymbol{u}^{b}$ ). All we need now is to generalize (14) by considering a distance vector between the centers of mass of the molecules $a$ and $b$, which does not necessarily lie in $x$-direction, but may be parallel to a unit vector $r$. This leads to

$$
\begin{aligned}
E_{a b}= & j_{2}\left[1-\left(u_{\lambda}^{a} u_{\lambda}^{b}\right)^{2}\right] \\
& +\left(j_{1}-j_{2}\right)\left[r_{\mu} u_{\mu}^{a} u_{v}^{a}-r_{\mathrm{\kappa}} u_{\mathrm{\kappa}}^{b} u_{\mathrm{v}}^{b}\right]\left[r_{\lambda} u_{\lambda}^{a} u_{\mathrm{v}}^{a}-r_{\sigma} u_{\sigma}^{b} u_{\mathrm{v}}^{b}\right] \\
& +\frac{1}{2}\left(j_{3}-j_{1}\right)\left[\left(r_{\mu} u_{\mu}^{a}\right)^{2}+\left(r_{\mathrm{v}} u_{\mathrm{v}}^{b}\right)^{2}\right]\left[1-\left(u_{\lambda}^{a} u_{\lambda}^{b}\right)^{2}\right] \\
= & j_{2}\left[1-\left(\boldsymbol{u}^{a} \cdot \boldsymbol{u}^{b}\right)^{2}\right]+\left(j_{1}-j_{2}\right) \\
& \cdot\left[\boldsymbol{r} \cdot \boldsymbol{u}^{a} \boldsymbol{r} \cdot \boldsymbol{u}^{a}+\boldsymbol{r} \cdot \boldsymbol{u}^{b} \boldsymbol{r} \cdot \boldsymbol{u}^{b}-2 \boldsymbol{r} \cdot \boldsymbol{u}^{a} \boldsymbol{r} \cdot \boldsymbol{u}^{b} \boldsymbol{u}^{a} \cdot \boldsymbol{u}^{b}\right] \\
& +\frac{1}{2}\left(j_{3}-j_{1}\right)\left[\left(\boldsymbol{r} \cdot \boldsymbol{u}^{a}\right)^{2}+\left(\boldsymbol{r} \cdot \boldsymbol{u}^{b}\right)^{2}\right]\left[1-\left(\boldsymbol{u}^{a} \cdot \boldsymbol{u}^{b}\right)^{2}\right] .
\end{aligned}
$$

Using $r_{\mu} r_{v}=\overleftrightarrow{\mathrm{r}_{\mu} \mathrm{r}_{\mathrm{v}}}+\frac{1}{3} \delta_{\mu v}$ we obtain

$$
\begin{aligned}
E_{a b}= & \frac{1}{3}\left(j_{1}+j_{2}+j_{3}\right)\left[1-\left(u_{\lambda}^{a} u_{\lambda}^{b}\right)^{2}\right] \\
& +\left(j_{1}-j_{2}\right) \overleftrightarrow{\mathrm{r}_{\mu} \mathrm{r}_{v}}\left[u_{\mu}^{a} u_{v}^{a}+u_{\mu}^{b} u_{v}^{b}-2 u_{\mu}^{a} u_{v}^{b} u_{\lambda}^{a} u_{\lambda}^{b}\right] \\
& +\frac{1}{2}\left(j_{3}-j_{1}\right) \overleftrightarrow{\mathrm{r}_{\mu} \mathrm{r}_{v}}\left[u_{\mu}^{a} u_{v}^{a}+u_{\mu}^{b} u_{v}^{b}\right]\left[1-\left(u_{\lambda}^{a} u_{\lambda}^{b}\right)^{2}\right] .
\end{aligned}
$$

The first term in (16) corresponds to the LebwohlLasher Hamilton. The remaining expressions are proportional to the anisotropic part of $r_{\mu} r_{v}$; thus, if we integrate $\boldsymbol{r}$ over a unit sphere keeping the two directors fixed, only the contribution of the LebwohlLasher term remains.

One expects, that $j_{1}, j_{2}$, and $j_{3}$ determine the strength of the splay-, twist- and the bend-contribution to the interaction energy. To investigate this point, we calculate $E_{a b}$ for some characteristical configurations of two molecules.

We introduce the abbreviations

$$
a=\boldsymbol{u}^{a} \cdot \boldsymbol{r}, \quad b=\boldsymbol{u}^{b} \cdot \boldsymbol{r} \quad \text { and } \quad c=\boldsymbol{u}^{a} \cdot \boldsymbol{u}^{b},
$$

so that (15) can be rewritten as

$$
\begin{aligned}
E_{a b}= & j_{2}\left(1-c^{2}\right)+\left(j_{1}-j_{2}\right)\left(a^{2}+b^{2}-2 a b c\right) \\
& +\frac{1}{2}\left(j_{3}-j_{1}\right)\left(a^{2}+b^{2}\right)\left(1-c^{2}\right) .
\end{aligned}
$$

Now we consider the following configurations, shown in Figure 12.

1) If the two molecules have parallel axes, we get $c=1$ and $a=b$, which leads to $E_{a b}=0$, independent of $\boldsymbol{r}$.

2) For $\boldsymbol{u}^{a} \perp \boldsymbol{u}^{b}$ and an $\boldsymbol{r}$, which is normal to the $\boldsymbol{u}^{a}, \boldsymbol{u}^{b}$ plain, $a, b$, and $c$ become 0 and $E_{a b}=j_{2}$. Since in this geometry we have a pure twist-deformation, $j_{2}$ is the coefficient for the twist energy contribution.

3) A configuration with $\boldsymbol{u}^{a} \perp \boldsymbol{u}^{b}$ and $\boldsymbol{r}$ in the $\boldsymbol{u}^{a}, \boldsymbol{u}^{b}$ plain includes no twist-deformation; the energy of this geometry consists of a splay- and a bend-contribution, while for symmetry reasons none of them predominates. In fact, with $c=0$ and $a^{2}+b^{2}=1$ we get $E_{a b}=\frac{1}{2}\left(j_{1}+j_{3}\right)$.

4) Finally we want to look at two configurations with an angle of $45^{\circ}$ between $\boldsymbol{u}^{a}$ and $\boldsymbol{r}$, which means $a=\frac{1}{\sqrt{2}}$. In the geometry 4) $\boldsymbol{u}^{b}$ is perpendicular to $\boldsymbol{r}$ and the configuration is rather splay-like. With $b=0$ and $c=\frac{1}{\sqrt{2}}$ we get $E_{a b}^{(4)}=\frac{1}{8}\left(3 j_{1}+j_{3}\right)$.

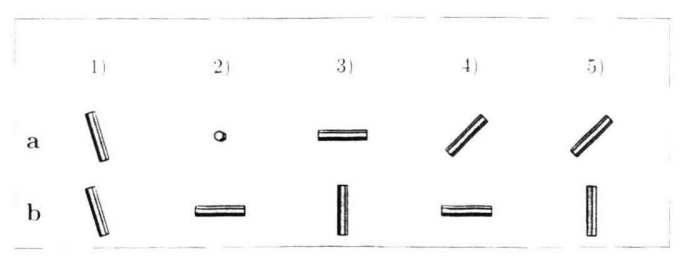

Fig. 12. Test configurations of the molecules $a$ and $b$ for the extended Lebwohl-Lasher model. 
5) For the rather bend-like geometry 5) with $\boldsymbol{u}^{b} \| \boldsymbol{r}$, which means $b=1$ and $c=\frac{1}{\sqrt{2}}$, we get $E_{a b}^{(5)}$ $=\frac{1}{8}\left(j_{1}+3 j_{3}\right)$. One obtains that $E_{a b}^{(4)}-E_{a b}^{(5)}$ $=\frac{1}{4}\left(j_{1}-j_{3}\right)$, which suggests that $j_{1}$ belongs to the splay energy, while $j_{3}$ belongs to the bend contribution.

It seems worthwhile to study the statistical properties of the introduced extension of the LebwohlLasher model. For example, one could investigate the temperature dependence of the three elastic coefficients near the critical temperature $T_{\mathrm{c}}$.

\section{Concluding Remarks}

We have presented a Monte Carlo method to produce equilibrium orientation fields for a nematic liquid crystal. The algorithm is based on the Frank free energy with three elastic coefficients and conserves the nematic symmetry, where $\boldsymbol{n}$ and $-\boldsymbol{n}$ are equivalent. All direct symmetrical discretisations of the spatial derivatives lead to uncoupled subsystems in the simulation; we needed to take the analytic average of four energy expressions based on asymmetric discretisations. Thus, especially with three elastic coefficients the calculations are rather time consuming.

[1] P. G. de Gennes, The Physics of Liquid Crystals, Clarendon Press, Oxford 1974.

[2] G. Vertogen and W. H. de Jeu, Thermotropic Liquid Crystals, Fundamentals, Springer, Berlin 1988.

[3] F. C. Frank, Discuss. Faraday Soc. 25, 19 (1958).

[4] F. Leenhouts and A. J. Dekker, J. Chem. Phys. 74, 1956 (1981).

[5] H. Gruler, Z. Naturforsch. 28a, 474 (1973).

[6] M. Schadt, Mol. Cryst. Liq. Cryst. 165, 405 (1988).

[7] M. A. Osipov and S. Hess, Molec. Phys. 78, 1191 (1993)

[8] A. Kilian and S. Hess, Liquid Crystals, Vol. 8, No. 4, 465 (1990).

[9] I. Pardowitz and S. Hess, Physica A 100, 540 (1980).

[10] W. Maier and A. Saupe, Z. Naturforsch. 14a, 882 (1959); 15a, 287 (1960).

[11] W. Muschik and C. Papenfuss, Physica A 201, 515 (1993).

[12] P. G. de Gennes, Mol. Cryst. Liq. Cryst. 12, 193 (1971).

[13] A. Kilian and S. Hess, Z. Naturforsch. 44a, 693 (1989).
Generally, against our original expectations we have to admit that the algorithm converges slower than simulations based on a relaxation equation for the alignment tensor [13]. Nevertheless, the Monte Carlo approach allows the use of the various advanced Monte Carlo techniques, which were developed to speed up the relaxation.

The described Monte Carlo algorithm can easily be extended to three dimensional calculations. Then one needs to average over eight Frank free energy expressions, built with each of the simple asymmetrical discretisations of the derivation term.

A comparison with the Lebwohl-Lasher model has revealed an analogy between the Hamilton of the latter and our discretized energy expression in the onecoefficient approximation. We have extended the Lebwohl-Lasher energy term to an expression, which distinguishes between splay-, twist- and bend-like configurations of neighboring molecules.

\section{Acknowledgements}

This work has been conducted under the auspices of the Sonderforschungsbereich SFB 335 "Anisotrope Fluide". Financial support by the DFG is gratefully acknowledged. We thank Dr. Axel Kilian and André Sonnet for helpful discussions.

[14] T. Gruhn, Diplomarbeit „Monte-Carlo-Untersuchungen der Ausrichtung nematischer Flüssigkristalle", Institut für Theoretische Physik, TU Berlin, 1995 (unpublished).

[15] M. P. Allen and D. J. Tildesley, Computer Simulation of Liquids, Clarendon Press, Oxford 1987.

[16] N. Metropolis, A. W. Rosenbluth, M. N. Rosenbluth, A. H. Teller, and E. Teller, J. Chem. Phys. 21, 1087 (1953).

[17] A. Kilian, Phys. Rev. E Vol. 50, 3774 (1994).

[18] D. W. Allender, G. P. Crawford, and J. W. Doane, Phys. Rev. Lett. 67, 1442 (1991).

[19] A. Sonnet, A. Kilian, and S. Hess, Phys. Rev. E 52, 718 (1995).

[20] P. A. Lebwohl and G. Lasher, Phys. Rev. A 6, 426 (1972).

[21] R. G. Priest, Mol. Cryst. Liq. Cryst. 17, 129 (1972)

[22] D. J. Cleaver and M. P. Allen, Phys. Rev. A 43, 1918 (1990). 